\title{
The efficacy and safety of cilostazol as an alternative to aspirin in Chinese patients with aspirin intolerance after coronary stent implantation: a combined clinical study and computational system pharmacology analysis
}

\author{
Ying XUE ${ }^{1,2, \# \text {, Zhi-wei FENG }}{ }^{2, \#}$, Xiao-ye $\mathrm{LI}^{1, \#}$, Zi-heng $\mathrm{HU}^{2}$, Qing $\mathrm{XU}^{1}$, Zi WANG ${ }^{3}$, Jia-hui CHENG ${ }^{4}$, Hong-tao SHI ${ }^{4}$, Qi-bing \\ $\mathrm{WANG}^{4}$, Hong-yi WU ${ }^{4, *}$, Xiang-Qun $\mathrm{XIE}^{2, *}$, Qian-zhou LV${ }^{1, *}$ \\ ${ }^{1}$ Department of Clinical Pharmacy, Zhongshan Hospital, Fudan University, Shanghai 200032, China; ${ }^{2}$ Department of Pharmaceutical \\ Sciences and Computational Chemical Genomics Screening Center, School of Pharmacy; NIH National Center of Excellence for \\ Computational Drug Abuse Research; Drug Discovery Institute; Departments of Computational Biology and Structural Biology, School \\ of Medicine, University of Pittsburgh, Pittsburgh, Pennsylvania, USA; ${ }^{3}$ School of Pharmacy, Fudan University, Shanghai 201203, China; \\ ${ }^{4}$ Department of Cardiology, Zhongshan Hospital, Fudan University, Shanghai 200032, China
}

\begin{abstract}
Dual antiplatelet therapy (DAT) with aspirin and clopidogrel is the standard regimen to achieve rapid platelet inhibition and prevent thrombotic events. Currently, little information is available regarding alternative antiplatelet therapy in patients with an allergy or intolerance to aspirin. Although cilostazol is already a common alternative to aspirin in clinical practice in China, its efficacy and safety remain to be determined. We retrospectively analyzed 613 Chinese patients who had undergone primary percutaneous coronary intervention (PCl). Among them, 405 patients received standard DAT (aspirin plus clopidogrel) and 205 patients were identified with intolerance to aspirin and received alternative DAT (cilostazol plus clopidogrel). There were no significant differences between the two groups in their baseline clinical characteristics. The main outcomes of the study included major adverse cardiac events (MACEs) and bleeding events during 12 months of follow-up. The MACEs endpoint was reached in 10 of 205 patients treated with cilostazol (4.9\%) and in 34 of 408 patients treated with aspirin (8.3\%). No statistically significant difference was observed in MACEs between the two groups. However, patients in the cilostazol group had less restenosis than did patients in the aspirin group (1.5\% vs $4.9 \%, P=0.035)$. The occurrence of bleeding events tended to be lower in the cilostazol group $(0.49 \%$ vs $2.7 \%, P=0.063)$. These clinical observations were further analyzed using network system pharmacology analysis, and the outcomes were consistent with clinical observations and preclinical data reports. We conclude that in Chinese patients with aspirin intolerance undergoing coronary stent implantation, the combination of clopidogrel with cilostazol may be an efficacious and safe alternative to the standard DAT regimen.
\end{abstract}

Keywords: dual antiplatelet therapy; aspirin; intolerance; cilostazol; percutaneous coronary intervention; major adverse cardiac events; network system pharmacology

Acta Pharmacologica Sinica (2018) 39: 205-212; doi: 10.1038/aps.2017.85; published online 21 Sep 2017

\section{Introduction}

Although drug-eluting stents (DES) have decreased the incidence of restenosis and repeat revascularization in patients undergoing percutaneous coronary intervention (PCI),

\footnotetext{
\#These authors contributed equally to this work.

${ }^{*}$ To whom correspondence should be addressed.

E-mail wu.hongyi@zs-hospital.sh.cn (Hong-yi WU); xix15@pitt.edu (Xiang-Qun XIE); Lv.qianzhou@zs-hospital.sh.cn (Qian-zhou LV)

Received 2017-02-27 Accepted 2017-05-19
}

excessive antiproliferative effects and hypersensitivity to DES have increased the risk of complicated thrombotic events ${ }^{[1-3]}$.

A combination of thienopyridine and aspirin is the standard dual antiplatelet therapy, which improves long-term clinical outcomes in the setting of PCI and acute coronary syndrome ${ }^{[4-6]}$. However, not all patients can be treated with these medications. Patients with a prior history of an aspirin "reaction" are routinely denied this medication, and reasons for aspirin "reaction" primarily include gastrointestinal intolerance, bleeding and allergy ${ }^{[7-10]}$. Currently, limited 
information is available regarding alternative antiplatelet therapy in patients with an intolerance to aspirin. Therefore, the optimal regimen of antiplatelet therapy in such patients has become a problem to be solved.

Cilostazol is a selective inhibitor of phosphodiesterase type III (PDE3) that inhibits platelet aggregation in response to adenosine diphosphate (ADP), epinephrine, collagen, and arachidonic acid ${ }^{[11]}$. It has been reported that the adjunctive use of cilostazol can overcome limitations of DES, with respect to both thrombosis and restenosis ${ }^{[12-14]}$. In addition, cilostazol is expected to have potential for achieving the critical balance between hemorrhagic risk and antiplatelet efficacy in patients with thrombotic disease and bleeding risk ${ }^{[15-16]}$. Hence, cilostazol is considered an alternative to aspirin in real-world clinical practice in China. However, its efficacy and safety remains to be determined, particularly for patients after coronary stent implantation ${ }^{[17]}$.

The present work aimed to study the efficacy and safety of cilostazol as an alternative to aspirin after coronary stent implantation in patients with intolerance to aspirin. The clinical observations were further analyzed by computational system pharmacology.

\section{Materials and methods \\ Study design and patients}

This study was a retrospective cohort analysis of 613 consecutive patients who underwent coronary stent implantation between July 2014 and July 2015 at Zhongshan Hospital. A total of 205 patients were identified with intolerance to aspirin and received alternative DAT. Our Medical Ethics Committee approved this study and waived the requirement for informed consent. Aspirin intolerance was defined as follows: i) a documented allergic reaction to aspirin; ii) patient's or referring physician's unwillingness to take or prescribe aspirin because of prior side effects and/or recurrent symptoms despite treatment with a proton pump inhibitor. Reasons for not taking aspirin included gastrointestinal intolerance (82.4\%), allergy $(6.8 \%)$, non-gastrointestinal bleeding $(2.9 \%)$ and other reasons $(7.8 \%)$. The aspirin-intolerant patients were treated with cilostazol in combination with clopidogrel as an alternative DAT regimen.

All patients had angina pectoris or a positive stress test and had native coronary artery lesions for which DES implantation was feasible. Patients were excluded if they had contraindications to clopidogrel or cilostazol; an age less than 18 years; a left ventricular ejection fraction $<30 \%$; a platelet count $<100000 / \mathrm{mm}^{3}$; severe liver and kidney insufficiency; or acute or chronic infection.

\section{Medication and $\mathrm{PCl}$ procedure}

All patients received drug-eluting stents and dual antiplatelet therapy. At least $24 \mathrm{~h}$ before the procedure, a loading dose of $300 \mathrm{mg}$ clopidogrel was administered, and a daily regimen of $75 \mathrm{mg}$ was prescribed for 12 months. Patients in the control group received a $300 \mathrm{mg}$ loading dose followed by a daily regimen of $100 \mathrm{mg}$. Patients in the study group received cilo- stazol (50 mg twice daily). The dose of cilostazol $(100 \mathrm{mg} / \mathrm{d})$ in the present study is the dose commonly used in our institution. Procedural details, including use of anticoagulation drugs and type of stents, were dependent upon the operators. All patients received a successful PCI.

\section{Clinical endpoints}

Major adverse cardiac events were defined as the composite of all-cause death, non-fatal myocardial infarction (MI), restenosis, target vessel revascularization (TVR) and stroke. Non-fatal myocardial infarction was defined as recurrent symptoms with new ST-segment elevation or re-elevation of cardiac markers to at least twice the upper limit of normal. TVR was defined as any repeat revascularization of the target vessel. Stroke was defined as persistent loss of neurological function developed after primary PCI and an acute lesion identified with magnetic resonance imaging (MRI). Bleeding was classified according to the Bleeding Academic Research Consortium (BARC) classification. Cases with a BARC score $>2$ were recorded ${ }^{[18]}$.

\section{Collection of demographic, medical, and laboratory data}

Demographic and medical data, including age, sex and comorbid conditions, were obtained from medical records or patient interviews. The body mass index (BMI) was calculated for each subject as the ratio of the weight in kilograms divided by the square of the height in meters. Blood pressure was measured in the supine position using a mercury sphygmomanometer with an appropriate cuff on the left arm; the average of two readings was used for the statistical analysis.

A 12-h overnight fasting venous blood sample was collected for measurements of hemoglobin, hematocrit, creatinine, alanine transaminase and platelets.

\section{CVDPlatform-cardiovascular disease (CVD)-specific chemogenomics database}

We have constructed a cardiovascular disease (CVD)-specific chemogenomics database ${ }^{[19]}$ that can be used for target/offtarget (or additional) identification and network system pharmacology analysis of small molecules and their potential targets. Several in-house chemoinformatics tools were also used, including TargetHunter, HTDocking, Blood-Brain Barrier (BBB) Predictor (developed by Xie's laboratory, Pittsburgh, PA, USA), etc ${ }^{[20-26]}$. CVDPlatform (www.cbligand.org/CVD) archived 984 CVD-related target proteins, 924 approved CVD drugs in clinical trials, 2080 active chemical compounds associated with the therapeutic targets of CVD, 276 cardiovascularrelated pathways, and 350765 references.

In the present work, we applied our CVDPlatform and HTDocking program to perform the network system pharmacological analysis of small molecules, including aspirin and cilostazol. Cytoscape 3.4.0 was used to generate, analyze and visualize the graphical network between targets and drug/ compounds, as described previously ${ }^{[19]}$.

\section{Statistical analysis}

Data are presented as the mean \pm SD for continuous variables 
and as the number and percentage for categorical variables. Normal distribution of continuous variables was confirmed using Kolmogorov-Smirnov testing. Comparisons of continuous variables between two groups were performed with Student's $t$-tests, and comparisons of categorical variables were assessed by Chi-square or Fisher's exact tests. Odds ratios (OR), confidence intervals (CI), and $P$-values from univariate regression analysis were presented.

Data were analyzed using IBM SPSS Statistics 19.0 software, and a $P<0.05$ was considered to indicate statistical significance.

\section{Results}

\section{Baseline characteristics}

Baseline clinical characteristics of the 613 patients are presented in Table 1. There were no significant differences in age, sex, or cardiovascular risk factors between the two groups. Medication profiles were not different between the two groups.

Lesion and procedural characteristics are shown in Table 2. The two groups had similar baseline angiographic and PCI procedural characteristics.

\section{Clinical outcomes}

Clinical follow-up for 12 months was obtained in all patients.
The clinical outcomes are summarized in Table 3. The efficacy endpoints, composite of death, MI, ischemic stroke, restenosis and TVR occurred in 10 patients $(4.9 \%)$ in the study group and 34 patients $(8.3 \%)$ in the control group, and the results were not significantly different (OR $0.56 ; 95 \% \mathrm{CI}=0.27-1.17 ; P=0.12$ ) (Table 3). MI, ischemic stroke and TVR also did not appear to differ significantly between the two study groups. However, the restenosis rate was statistically lower in groups treated with cilostazol than the control group $(1.5 \%$ vs $4.9 \%$; OR 0.29 ; 95\% CI=0.09-0.98; $P=0.035)$. The incidence of bleeding tended to be lower in the study group $(0.49 \%$ vs $2.7 \%$; OR $0.18 ; 95 \% \mathrm{CI}$ $=0.02-1.38 ; P=0.063)$.

\section{Network systems pharmacology studies}

We applied our constructed CVDPlatform (www.cbligand. org/CVD) and our network system pharmacology approach to predict and analyze the complex interactions between aspirin/cilostazol and cardiovascular-related target proteins. Our high-throughput molecular docking (www.CBLigand.org/ HTdocking) approach was used to map out the predicted interactions between aspirin/cilostazol and 984 target proteins in CVDPlatform. Briefly, the drug molecules were docked into the pocket of the target protein by using AutoDock Vina. We then ranked the potential cardiovascular therapeutic protein

Table 1. Demographic and clinical characteristics of the study participants.

\begin{tabular}{|c|c|c|c|}
\hline & Cilostazol $(n=205)$ & Aspirin $(n=408)$ & $P$ value \\
\hline Age (year) & $63.9 \pm 10.2$ & $62.9 \pm 8.8$ & 0.242 \\
\hline Men & $161(78.5 \%)$ & $331(81.1 \%)$ & 0.447 \\
\hline $\mathrm{BMI}\left(\mathrm{kg} / \mathrm{m}^{2}\right)$ & $25.1 \pm 2.6$ & $25.0 \pm 2.8$ & 0.946 \\
\hline Hypertension (\%) & $133(64.9 \%)$ & $258(63.2 \%)$ & 0.690 \\
\hline Hyperlipoidemia (\%) & $11(16.0 \%)$ & $28(16.0 \%)$ & 0.474 \\
\hline Diabetes (\%) & $56(27.3 \%)$ & $115(28.2 \%)$ & 0.821 \\
\hline Stroke $(\%)$ & $11(5.4 \%)$ & $24(5.9 \%)$ & 0.795 \\
\hline Current smoker (\%) & 79 (38.5\%) & $170(41.7 \%)$ & 0.457 \\
\hline Previous percutaneous coronary intervention & $55(26.8 \%)$ & $101(24.8 \%)$ & 0.625 \\
\hline \multicolumn{4}{|l|}{ Clinical diagnosis } \\
\hline Stable angina & $139(67.8 \%)$ & $272(66.7 \%)$ & 0.777 \\
\hline ACS & $66(32.2 \%)$ & $136(33.3 \%)$ & 0.777 \\
\hline Systolic BP (mmHg) & $128.2 \pm 14.1$ & $126.3 \pm 16.8$ & 0.158 \\
\hline Diastolic BP (mmHg) & $77.5 \pm 9.2$ & $76.4 \pm 9.6$ & 0.213 \\
\hline ALT & $30.4 \pm 28.4$ & $31.7 \pm 25.6$ & 0.602 \\
\hline Creatinine $(\mu \mathrm{mol} / \mathrm{L})$ & $80.1 \pm 21.1$ & $82.0 \pm 46.4$ & 0.585 \\
\hline Hemoglobin (g/L) & $134.3 \pm 22.1$ & $131.1 \pm 25.6$ & 0.134 \\
\hline Hematocrit (\%) & $40.0 \pm 4.2$ & $39.2 \pm 7.7$ & 0.159 \\
\hline $\operatorname{PLT}\left(\times 10^{9} / \mathrm{L}\right)$ & $206.1 \pm 71.8$ & $206.1 \pm 72.1$ & 0.999 \\
\hline LVEF (\%) & $61.9 \pm 8.7$ & $61.0 \pm 8.8$ & 0.28 \\
\hline \multicolumn{4}{|l|}{ Medication } \\
\hline Clopidogrel & 205 (100\%) & $408(100 \%)$ & 1.00 \\
\hline GP Ilb/IIla & 39 (19.0\%) & 82 (20.1\%) & 0.753 \\
\hline Statins & 199 (97.1\%) & 394 (96.6\%) & 0.881 \\
\hline$\beta$-blocker & $164(80.0 \%)$ & 327 (80.1\%) & 0.827 \\
\hline ACEI/ARB & $133(64.9 \%)$ & $270(66.2 \%)$ & 0.659 \\
\hline Nitrates & 119 (58.0\%) & 237 (58.1\%) & 0.911 \\
\hline
\end{tabular}

BMI, Body Mass Index; ALT, Alanine Transaminase; PLT, Platelet; LVEF, Left Ventricular Ejection Fraction; GP IIb/IIla, Glycoprotein IIb/IIla; ACEI, Angiotensin Converting Enzyme Inhibitors; ARB, Angiotensin Receptor Blocker. 
Table 2. Angiographic characteristics and procedural results.

\begin{tabular}{lll}
\hline & Cilostazol $(n=205)$ & Aspirin $(n=408)$ \\
\hline Lesion locations & & $P$ value \\
Left anterior descending coronary artery & $110(53.9 \%)$ & $236(57.8 \%)$ \\
Left circumflex artery & $57(27.7 \%)$ & $107(26.2 \%)$ \\
Right coronary artery & $66(32.0 \%)$ & $113(27.7 \%)$ \\
Left main coronary artery & $9(4.4 \%)$ & $20(4.4 \%)$ \\
Number of diseased vessels: & & $186(45.6 \%)$ \\
1 & $102(49.8 \%)$ & $139(34.0 \%)$ \\
2 & $65(31.6 \%)$ & $83(20.4 \%)$ \\
3 & $38(18.4 \%)$ & $1.60 \pm 0.77$ \\
Number of stents & $1.64 \pm 0.84$ & $41.0 \pm 22.6$ \\
Total stent length $(\mathrm{mm})$ & $43.3 \pm 27.4$ & 0.67 \\
\hline
\end{tabular}

Table 3. 12-Month clinical outcomes.

\begin{tabular}{|c|c|c|c|c|c|}
\hline & Cilostazol $(n=205)$ & Aspirin $(n=408)$ & $P$ value & OR & $95 \% \mathrm{Cl}$ \\
\hline $\operatorname{MACE}[n(\%)]$ & $10(4.9 \%)$ & $34(8.3 \%)$ & 0.118 & 0.56 & $0.27-1.17$ \\
\hline Cardiac death & $2(0.98 \%)$ & $3(0.74 \%)$ & 0.755 & 1.33 & $0.22-7.81$ \\
\hline Myocardial infarction & $4(1.9 \%)$ & $14(3.4 \%)$ & 0.306 & 0.56 & $0.18-1.72$ \\
\hline Stroke $[n(\%)]$ & $1(0.49 \%)$ & 0 & 0.158 & - & - \\
\hline
\end{tabular}

targets to specific drug according to the docking scores. Top listed targets with higher docking scores may have higher binding affinities or a greater chance of interacting with our input compounds. Subsequently, we mapped out a pharmacological network of interactions between drug compounds and target proteins at the molecular level.

As shown in Figure 1, the potential (or additional) target proteins of these two drugs were predicted and ranked on the basis of docking scores (cut-off value: 6.5) with the interaction network. Interestingly, our results showed that most of the docking scores of target proteins for cilostazol were higher than that for aspirin (Table 4). This result is reasonable because cilostazol fits better into the pockets of target proteins, owing to its flexible structure and stronger interactions. Moreover, our predicted results correlate well with the known therapeutic targets of aspirin and cilostazol (higher docking scores). For example, prostaglandin $\mathrm{G} / \mathrm{H}$ synthase 1 /prostaglandin $\mathrm{G} / \mathrm{H}$ synthase 2 (gene name: COX1/COX2), cytochrome P450 1A2 (gene name: CYP1A2), and serum albumin (gene name: ALB) ranked first/twelfth, fourth and sixth, respectively, in the list of aspirin target proteins. Several recent literature reports have found that the the $\mathrm{IC}_{50}$ for aspirin toward COX1/COX2 is $0.3 / 2.4 \mu \mathrm{mol} / \mathrm{L}$, respectively ${ }^{[27-28]}$. The docking scores (mean value from two independent docking runs) for aspirin toward COX1 and COX2 showed differences in our computational work, in which the docking scores of COX1 and COX2 were 8.1 and 6.7 , respectively, thus indicat- ing that the docking study was sufficiently sensitive to reflect their different binding affinities. cGMP-inhibited $3^{\prime}, 5^{\prime}$-cyclic phosphodiesterase A (gene name: PDE3A) ranked eleventh in the list of cilostazol target proteins. Three additional shared targets for these two drugs were also validated by bioassays, including angiotensin II type-2 (AT2) receptor (gene name: AGTR2), angiotensin-converting enzyme (gene name: ACE), and cathepsin G (gene name: CTSG).

\section{Discussion}

To the best of our knowledge, this study is the first to investigate the efficacy and safety of cilostazol as a substitute for aspirin. In the present work, we performed a retrospective study to analyze the clinical outcomes in 205 of 613 patients undergoing coronary stent implantation who were treated with cilostazol as an alternative antiplatelet agent to aspirin. The statistical analysis results revealed that cilostazol, compared with aspirin, has the potential to decrease restenosis events, and the occurrence of bleeding events tended to be lower in the cilostazol group. No significant difference was observed in MACEs between the two groups. The main finding of the present study was that in aspirin-intolerant patients undergoing coronary stent implantation, the combination of cilostazol with clopidogrel appeared well tolerated and effective.

To better understand the outcome analysis results of the clinical observation above, we carried out a molecular network system pharmacology (MSP) investigation by using our estab- 


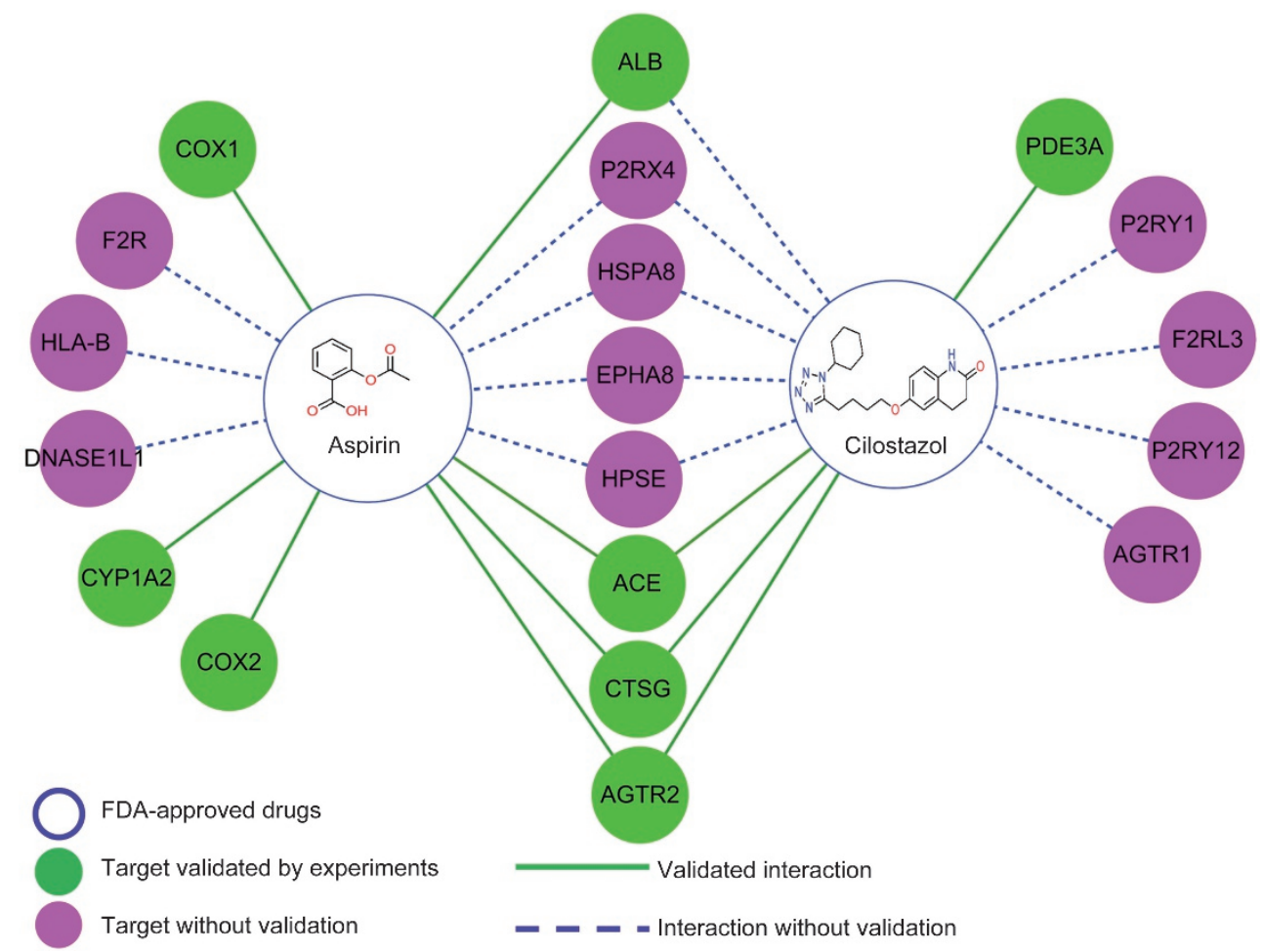

Figure 1. Network system pharmacology analysis of aspirin/cilostazol and the predicted targets by using our established CVDPlatform and HTDocking target identification program.

Table 4. The predicted protein targets for aspirin and cilostazol using HTDocking Program (Docking Score represents -log $K_{\mathrm{d}}$ ).

\begin{tabular}{|c|c|c|c|}
\hline Protein & Docking score & Drug name & Gene name \\
\hline Prostaglandin G/H synthase 1 & 8.1 & Aspirin & cox1 \\
\hline HLA class I histocompatibility antigen, B-39 alpha chain & 7.8 & Aspirin & HLA-B \\
\hline Deoxyribonuclease-1-like 1 & 7.6 & Aspirin & DNASE1L1 \\
\hline Cytochrome P450 1A2 & 7.5 & Aspirin & CYP1A2 \\
\hline Heparanase & 7.3 & Aspirin & HPSE \\
\hline Serum albumin & 7.2 & Aspirin & ALB \\
\hline Ephrin type-A receptor 8 & 7.2 & Aspirin & EPHA8 \\
\hline Heat shock cognate $71 \mathrm{kDa}$ protein & 7.1 & Aspirin & HSPA8 \\
\hline Proteinase-activated receptor 1 (PAR1) & 7.0 & Aspirin & $\mathrm{F} 2 \mathrm{R}$ \\
\hline P2X purinoceptor 4 (P2X4) & 6.9 & Aspirin & $\mathrm{P} 2 \mathrm{RX} 4$ \\
\hline Angiotensin II type-2 (AT2) receptor & 6.8 & Aspirin & AGTR2 \\
\hline Prostaglandin G/H synthase 2 & 6.7 & Aspirin & $\operatorname{cox} 2$ \\
\hline Cathepsin G & 6.6 & Aspirin & CTSG \\
\hline Angiotensin-converting enzyme & 6.5 & Aspirin & ACE \\
\hline P2Y purinoceptor 1 (P2Y1) & 11.8 & Cilostazol & P2RY1 \\
\hline Heparanase & 11.5 & Cilostazol & HPSE \\
\hline Ephrin type-A receptor 8 & 11.1 & Cilostazol & EPHA8 \\
\hline Cathepsin G & 11.0 & Cilostazol & CTSG \\
\hline Serum albumin & 10.9 & Cilostazol & ALB \\
\hline Heat shock cognate $71 \mathrm{kDa}$ protein & 10.9 & Cilostazol & HSPA8 \\
\hline Angiotensin II type-2 (AT2) receptor & 10.9 & Cilostazol & AGTR2 \\
\hline P2X purinoceptor 4 (P2X4) & 10.8 & Cilostazol & P2RX4 \\
\hline Angiotensin-converting enzyme & 10.6 & Cilostazol & ACE \\
\hline Angiotensin II Type-1 (AT1) receptor & 10.1 & Cilostazol & AGTR1 \\
\hline cGMP-inhibited 3',5'-cyclic phosphodiesterase A & 9.8 & Cilostazol & PDE3A \\
\hline Proteinase-activated receptor 4 (PAR4) & 9.7 & Cilostazol & F2RL3 \\
\hline P2Y purinoceptor 12 (P2Y12) & 9.4 & Cilostazol & P2RY12 \\
\hline
\end{tabular}


lished cardiovascular disease-domain specific chemogenomics knowledgebase, TargetHunter and HTDocking programs. We analyzed the complex interactions of aspirin and cilostazol with cardiovascular-related target proteins in biological systems. The results were consistent with clinical observations, showing that cilostazol has additional protein targets that may explain its superior effect on prevention of restenosis and lower risk of bleeding. The combined clinical data-mining and system pharmacology studies also provide a promising approach to better understand the outcomes of cilostazol as an alternative antiplatelet agent to aspirin at both the clinical and molecular levels.

Although the combination of aspirin and one of the P2Y purinoceptor 12 (P2Y12) inhibitors (such as clopidogrel) is recommended as standard dual antiplatelet therapy after percutaneous coronary intervention, patients with a prior history of an aspirin "reaction" are routinely denied this standard DAT. Several studies have suggested the need for an alternative regimen for patients with aspirin intolerance ${ }^{[29-30]}$. Thus, an optimal regimen of antiplatelet therapy for those patients is continually evolving.

Cilostazol is a cAMP PDE-III inhibitor that exerts a strong antithrombotic effect not only by directly inhibiting platelet function but also by decreasing the number of activated circulating platelets while maintaining a minimal risk of bleeding $^{[15,31-32]}$. In Asian countries, cilostazol is widely used as an antiplatelet agent for the secondary prevention of ischemic events, and it is often preferred over aspirin or thienopyridines, owing to its comparable efficacy and more favorable safety profile ${ }^{[15,33]}$. In fact, cilostazol has been shown to be associated with decreased bleeding risk, as compared with other antiplatelet agents. In addition, cilostazol also decreases neointimal proliferative reactions after coronary stent implantation $^{[14,34-40]}$. In clinical studies, cilostazol-based triple antiplatelet treatment (TAPT) has been found to have the potential to decrease major adverse cardiac events and stent thrombosis without increasing the risk of bleeding ${ }^{[33-36,40]}$.

The known pharmacological action of cilostazol on inhibiting platelet activation and aggregation, as well as the proven anti-ischemic effects demonstrated above, has provided a rationale for clinicians to use cilostazol after stent implantation in the rare subset of patients who are intolerant or allergic to aspirin. However, in clinical practice, little information is available on whether cilostazol may be a successful alternative antiplatelet therapy for these patients.

Our retrospective study demonstrated that cilostazol has comparable efficacy to aspirin in preventing MACEs after stent implantation. It has been reported that cilostazol, compared with aspirin, has the potential to decrease restenosis after balloon angioplasty, stent implantation, and directional coronary atherectomy ${ }^{[12-14,41-42]}$. In line with results from previous studies, in this study, the use of cilostazol, compared with aspirin, resulted in a lower rate of restenosis in coronary artery disease patients. Patients with a prior history of aspirin intolerance are often at higher risk of gastrointestinal bleeding. Our results suggested that cilostazol, as an alternative for aspirin- intolerant patients, did not increase the risk of bleeding $(0.49 \%$ in cilostazol group vs $2.7 \%$ in the aspirin group, $P=0.063$ ).

Using a molecular systems pharmacology approach, we mapped out the potential molecular targets for aspirin and cilostazol in addition to those known drug targets. The system pharmacology predictions were consistent with clinical observations and preclinical data reports. A multi-target drug is a compound that can modulate multiple proteins. For example, after aspirin molecules enter the cell, most will act on major targets (for example, COX1 or COX2). However, others may also act on other targets (ie, off-target effects), which may generate either therapeutic effects or side effects. Thus, computational system pharmacology studies can be used to identify other potential CVD-related targets associated with aspirin, in addition to COX1/COX2, and with cilostazol, in addition to PDE3A. Importantly, our system pharmacology predictions indicated that cilostazol showed synergetic benefits for: (1) anti-bleeding effect by acting on the angiotensin II type-1 (AT1) receptor (gene name: AGTR1) and (2) prevention of restenosis by targeting proteinase-activated receptor 4 (PAR4) (gene name: F2RL3) and/or P2Y purinoceptor 12 (P2Y12) (gene name: P2RY12), which are among the top predicted targets for cilostazol (Figure 1, P2Y12 PDB: 4NTJ) ${ }^{[43]}$. Schindler et al have reported that, using the AT1-receptor antagonist candesartan in patients with severe atherosclerosis after superficial femoral artery stenting produces a significantly better effect against restenosis than the treatment with the ACE inhibitor quinapril ${ }^{[44]}$. Goel et al have found that Ticagrelor, a P2Y12 antagonist, prevents restenosis by inhibiting the inflammation induced by the P2Y12 receptor ${ }^{[45]}$. Wong et al have shown that PAR4 inhibition has the potential therapeutic advantage of antithrombotic activity with a low bleeding risk ${ }^{[46]}$. Interestingly, our predictions have revealed that proteinase-activated receptor 1 (PAR1) (gene name: F2R) is one of the potential targets of aspirin, whereas PAR1 inhibitors/antagonists appear to increase the risk of bleeding significantly $^{[47]}$. Further experiments are required to validate these predictions. Therefore, the clinical studies performed in this work combined with computational system pharmacology mapping should be meaningful for assessing the therapeutic potential of cilostazol as an aspirin alternative.

There are several limitations of this study. First, this was a single-center observational study, and the patient population was relatively limited. We could not attempt to justify the sample size, because there were no available data regarding cilostazol as an alternative to aspirin; moreover, patients who are intolerant to aspirin are uncommon. Second, we could not administer a higher dose of cilostazol $(200 \mathrm{mg} / \mathrm{d})$, because some patients may not tolerate that dose. The dose of cilostazol $(100 \mathrm{mg} / \mathrm{d})$ in the present study is the dose commonly used in our institution. Third, dual antiplatelet therapy with thienopyridine and aspirin is a standard treatment, whereas the use of cilostazol instead of aspirin is not a generally accepted treatment at present. Finally, because clopidogrelPPI interaction is an ongoing controversy, we did not consider the influence of PPI. In our previous study ${ }^{[48]}$, we have found 
that the efficacy of clopidogrel is not associated with PPI use. As a result, to confirm that the methodology is a good approach for a particular group of patients would require extensive studies involving a large number of patient populations; we are addressing this goal in ongoing efforts. Regardless of these limitations, our studies compared cilostazol with aspirin, in contrast to previous studies, which have focused on the additional inhibition of platelet aggregation by cilostazol when co-administered with aspirin and clopidogrel ${ }^{[44-47]}$. Therefore, these data warrant a larger scale prospective randomized controlled trial for patients with aspirin intolerance.

In conclusion, in this cohort of patients with aspirin intolerance undergoing coronary stent implantation, cilostazol was well tolerated and effective. Our results suggest that cilostazol may be a reasonable alternative to aspirin in patients after stent implantation.

\section{Disclosures}

Dr Xiang-Qun XIE serves as a consultant for Oxis Biotech under an agreement with the University of Pittsburgh.

\section{Acknowledgements}

This study was supported by the project of Shanghai Key Specialty Clinical Pharmacy and China Scholarship Council (awarded to Ying XUE). The authors would like to acknowledge funding support from the NIH (No P30 DA035778) awarded to Xie's laboratory).

\section{Author contribution}

Xiang-Qun XIE, Hong-yi WU, Qian-zhou LV, and Ying XUE designed research; Ying XUE, Zhi-wei FENG, Xiaoye LI, Qing XU, Zi WANG, Jia-hui CHEN, Hong-tao SHI and Qi-bing WANG performed research; Xiang-Qun XIE, Ying XUE, Xiao-ye LI and Zi-heng HU contributed new analytical tools and reagents; Ying XUE, Zhi-wei FENG and Zi-heng WU analyzed data; Ying XUE and Zhi-wei FENG wrote the paper.

\section{References}

1 Solinas E, Dangas G, Kirtane AJ, Lansky AJ, Franklin-Bond T, Boland P, et al. Angiographic patterns of drug-eluting stent restenosis and oneyear out-comes after treatment with repeated percutaneous coronary intervention. Am J Cardiol 2008; 102: 311-5.

2 Daemen J, Wenaweser P, Tsuchida K, Ab-recht L, Vaina S, Morger C, et al. Early and late coronary stent thrombosis of sirolimus-eluting and paclitaxel-eluting stents in routine clinical practice: data from a large two-institutional cohort study. Lancet 2007; 369: 667-78.

3 Mauri L, Hsieh WH, Massaro JM, Ho KK, D'Agostino R, Cutlip DE. Stent thrombosis in randomized clinical trials of drug-eluting stents. N Engl J Med 2007; 356: 1020-9.

4 Chen ZM, Jiang LX, Chen YP, Xie JX, Pan HC, Peto R, et al. Addition of clopidogrel to aspirin in 45,852 patients with acute myocardial infarction: randomised placebo-controlled trial. Lancet 2005; 366: 1607-21.

5 Sabatine MS, Cannon CP, Gibson CM, López-Sendón JL, Montalescot $\mathrm{G}$, Theroux P, et al. Addition of clopidogrel to aspirin and fibrinolytic therapy for myocardial infarction with ST-segment elevation. N Engl J
Med 2005; 352: 1179-89.

6 Cohen M. Antiplatelet therapy in percutaneous coronary intervention: a critical review of the $2007 \mathrm{AHA} / \mathrm{ACC} / \mathrm{SCAl}$ guidelines and beyond. Catheter Cardiovasc Interv 2009; 74: 579-97.

7 Feng $\mathrm{CH}$, White AA, Stevenson DD. Characterization of aspirin allergies in patients with coronary artery disease. Ann Allergy Asthma Immunol 2013; 110: 92-5.

8 Newby LK, Bhapkar MV, White HD. Aspirin use post-acute coronary syndromes: intolerance, bleeding and discontinuation. J Thromb Thrombolysis 2003; 16: 119-28.

9 Latib A, lelasi A, Ferri L, Chieffo A, Godino C, Carlino M, et al. Aspirin intolerance and the need for dual antiplatelet therapy after stent implantation: A proposed alternative regimen. Int J Cardiol 2013; 165: 444-7.

10 Cook KA, White AA. Rapid aspirin challenge in patients with aspirin allergy and acute coronary syndromes. Curr Allergy Asthma Rep 2016; 16: 11.

11 Cone J, Wang S, Tandon N, Fong M, Sun B, Sakurai K, et al. Comparison of the effects of cilostazol and milrinone on intracellular CAMP levels and cellular function in platelets and cardiac cells. J Cardiovasc Pharmacol 1999; 34: 497-504

12 Guerra E1, Byrne RA, Kastrati A. Pharmacological inhibition of coronary restenosis: systemic and local approaches. Expert Opin Pharmacother 2014; 15: 2155-71.

13 Jang JS, Jin HY, Seo JS, Yang TH, Kim DK, Kim DS, et al. A metaanalysis of randomized controlled trials appraising the efficacy and safety of cilostazol after coronary artery stent implantation. Cardiology 2012; 122: 133-43.

14 Lee SW, Park SW, Kim YH, Yun SC, Park DW, Lee CW, et al. Comparison of triple versus dual antiplatelet therapy after drugeluting stent implantation (from the DECLARE-Long trial). Am J Cardiol 2007; 100: 1103-8.

15 Goto S. Cilostazol: potential mechanism of action for anti-thrombotic effects accompanied by a low rate of bleeding. Atheroscler Suppl 2005; 6: 3-11.

16 Takeuchi K, Takayama S, Izuhara C. Comparative effects of the antiplatelet drugs, clopidogrel, ticlopidine, and cilostazol on aspirininduced gastric bleeding and damage in rats. Life Sci 2014; 110: 77-85.

17 Porto I, D'Amario D, Crea F. Cilostazol and primary-PCl: mirage or good alternative? Curr Vasc Pharmacol 2012; 10: 468-71.

18 Matic DM, Milasinovic DG, Asanin MR, Mrdovic IB, Marinkovic JM, Kocev NI, et al. Prognostic implications of bleeding measured by Bleeding Academic Research Consortium (BARC) categorisation in patients undergoing primary percutaneous coronary intervention. Heart 2014; 100: 146-52.

19 Zhang H, Ma S, Feng Z, Wang D, Li C, Cao Y, et al. Cardiovascular disease chemogenomics knowledgebase-guided target identification and drug synergy mechanism study of an herbal formula. Sci Rep 2016; 28: 6

20 Zhang Y, Wang L, Feng Z, Cheng H, McGuire TF, Ding Y, et al. StemCellCKB: an integrated stem cell-specific chemogenomics knowledgebase for target identification and systems-pharmacology research. J Chem Inf Model 2016; 56: 1995-2004

21 Liu H, Wang L, Lv M, Pei R, Li P, Pei Z, et al. AlzPlatform: an Alzheimer's disease domain-specific chemogenomics knowledgebase for polypharmacology and target identification research. J Chem Inf Model 2014; 54: 1050-60

22 Feng Z, Pearce LV, Xu X, Yang X, Yang P, Blumberg PM, et al. Structural insight into tetrameric hTRPV1 from homology modeling, molecular docking, molecular dynamics simulation, virtual screening, 
and bioassay validations. J Chem Inf Model 2015; 55: 572-88.

23 Ma C, Wang L, Xie XQ. Ligand classifier of adaptively boosting ensemble decision stumps (LiCABEDS) and its application on modeling ligand functionality for 5-HT-subtype GPCR families. J Chem Inf Model 2011; 51: 521-31.

24 Ma C, Wang L, Xie XQ. GPU accelerated chemical similarity calculation for compound library comparison. J Chem Inf Model 2011; 51: 1521-7.

25 Wang L, Ma C, Wipf P, Liu H, Su W, Xie XQ. TargetHunter: an in silico target identification tool for predicting therapeutic potential of small organic molecules based on chemogenomic database. AAPS J 2013; 15: 395-406.

26 Xu X, Ma S, Feng Z, Hu G, Wang L, Xie XQ. Chemogenomics knowledgebase and systems pharmacology for hallucinogen target identification-Salvinorin A as a case study. J Mol Graph Model 2016; 70: 284-95.

27 Abdur Rahim M, Praveen Rao PN, Bhardwaj A, Kaur J, Huang Z, Knaus EE. Isomeric acetoxy analogs of celecoxib and their evaluation as cyclooxygenase inhibitors. Bioorg Med Chem Lett 2011; 21: 607480.

28 Abdellatif KR, Chowdhury MA, Dong Y, Das D, Yu G, Velázquez CA, et al. Dinitroglyceryl and diazen-1-ium-1,2-diolated nitric oxide donor ester prodrugs of aspirin, indomethacin and ibuprofen: synthesis, biological evaluation and nitric oxide release studies. Bioorg Med Chem Lett 2009; 19: 3014-8.

29 Latib A, Ielasi A, Ferri L, Chieffo A, Godino C, Carlino M, et al. Aspirin intolerance and the need for dual antiplatelet therapy after stent implantation: a proposed alternative regimen. Int J Cardiol 2013; 165: 444-7.

30 Agarwal SK. Single antiplatelet therapy after percutaneous coronary intervention in patients allergic to aspirin. Cardiovasc Revasc Med 2014; 15: 308-10.

31 Meadows TA, Bhatt DL. Clinical aspects of platelet inhibitors and thrombus formation. Circ Res 2007; 100: 1261-75.

32 Sallustio F, Rotondo F, Di Legge S, Stanzione P. Cilostazol in the management of atherosclerosis. Curr Vasc Pharmacol 2010; 8: 36372.

33 Lee SW, Park SW, Hong MK, Kim YH, Lee BK, Song JM, et al. Triple versus dual antiplatelet therapy after coronary stenting: impact on stent thrombosis. J Am Coll Cardiol 2005; 46: 1833-7.

34 Lee SW, Park SW, Yun SC, Kim YH, Park DW, Kim WJ, et al. Triple antiplatelet therapy reduces ischemic events after drug-eluting stent implantation: rug-luting stenting followed by cilostazol treatment reduces adverse serious cardiac Events (DECREASE registry). Am Heart J 2010; 159: 284-91.

35 Han Y, Li Y, Wang S, Jing Q, Wang Z, Wang D, et al. Cilostazol in addition to aspirin and clopidogrel improves long-term outcomes after percutaneous coronary intervention in patients with acute coronary syndromes: a randomized, controlled study. Am Heart J 2009; 157: 733-9.

36 Biondi-Zoccai GGL, Lotrionte M, Anselmino M, Moretti C, Agostoni P, Testa L, et al. Systematic review and meta-analysis of randomized clinical trials appraising the impact of cilostazol after percutaneous coronary intervention. Am Heart J 2008; 155: 1081-9.

37 Douglas JS Jr. Role of adjunct pharmacologic therapy in the era of drug-eluting stents. Atheroscler Suppl 2005; 6: 47-52.

38 Douglas JS, Holmes DR, Kereiakes DJ, Grines CL, Block E, Ghazzal ZM, et al. Cilostazol for Restenosis Trial (CREST) Investigators. Coronary stent restenosis in patients treated with cilostazol. Circulation 2005; 112: 2826-32.

39 Park SW, Lee CW, Kim HS, Lee NH, Nah DY, Hong MK, et al. Effects of cilostazol on angiographic restenosis after coronary stent placement. Am J Cardiol 2000; 86: 499-503.

40 Lee S W, Park S W, Kim Y H, Yun S C, Park D W, Lee C W, et al. Drug-eluting stenting followed by cilostazol treatment reduces late restenosis in patients with diabetes mellitus the DECLARE-DIABETES Trial (a randomized comparison of triple antiplatelet therapy with dual antiplatelet therapy after drug-eluting stent implantation in diabetic patients). J Am Coll Cardiol 2008; 51: 1181-7.

41 Kunishima T, Musha H, Eto F, Iwasaki T, Nagashima J, Masui Y, et al. A randomized trial of aspirin versus cilostazol therapy after successful coronary stent implantation. Clin Ther 1997; 19: 1058-66.

42 Take S, Matsutani M, Ueda H, Hamaguchi H, Konishi H, Baba Y, et al. Effect of cilostazol in preventing restenosis after percutaneous transluminal coronary angioplasty. Am J Cardiol 1997; 79: 1097-9.

43 Zhang K, Zhang J, Gao ZG, Zhang D, Zhu L, Han GW, et al. Structure of the human $\mathrm{P} 2 \mathrm{Y} 12$ receptor in complex with an antithrombotic drug. Nature 2014; 509: 115-8.

44 Schindler C, Mueller A, Bramlage P, Boecking W, Kirch W, Schweizer J. Comparison of selective AT1-receptor blockade versus ACE inhibition for restenosis prophylaxis in patients with peripheral occlusive arterial disease after stent angioplasty: a randomized, controlled, proof-ofconcept study. Angiology 2007; 58: 710-6.

45 Goel D. Ticagrelor: The first approved reversible oral antiplatelet agent. Int J Appl Basic Med Res 2013; 3: 19-21.

46 Wong PC, Seiffert D, Bird JE, Watson CA, Bostwick JS, Giancarli M, et al. Blockade of protease-activated receptor-4 (PAR4) provides robust antithrombotic activity with low bleeding. Sci Transl Med 2017; 9: 371.

47 Chatterjee S, Sharma A, Mukherjee D. PAR-1 antagonists: current state of evidence. J Thromb Thrombolysis 2013; 35: 1-9.

48 Wu H, Qian J, Xu J, Sun A, Sun W, Wang Q, et al. Effects of CYP2C19 variant alleles on postclopidogrel platelet reactivity and clinical outcomes in an actual clinical setting in China. Pharmacogenet Genomics 2012; 22: 887-90. 